\title{
Proteomic (antibody microarray) exploration of the molecular mechanism of action of the specific COX-2 inhibitor DuP 697
}

\author{
VIJAY AGARWAL ${ }^{1,2,3^{*}}$, VICTORIA C. HODGKINSON ${ }^{1^{*}}$, GINA L. EAGLE ${ }^{1}$, \\ LUCY SCAIFE $^{1}$, MICHAEL J. LIND ${ }^{1,2,3}$ and LYNN CAWKWELL ${ }^{1,2}$ \\ ${ }^{1}$ Cancer Biology Proteomics Group, Postgraduate Medical Institute of the University of Hull; ${ }^{2}$ Hull York Medical School;
${ }^{3}$ Queens Centre for Oncology and Haematology, Hull and East Yorkshire NHS Trust, Hull, UK
}

Received October 9, 2012; Accepted December 7, 2012

DOI: $10.3892 /$ ijo.2013.1784

\begin{abstract}
We have previously shown that specific COX-2 inhibitors, including DuP 697, have anti-proliferative effects on mesothelioma cells and potentiate the cytotoxicity of pemetrexed. Here, we used a novel proteomic approach to explore the mechanism of action of this agent. COX-2-positive cell lines MSTO-211H (mesothelioma) and A549 (lung cancer) were exposed to DuP 697 for 72 h. Drug carrier only was added to control cells. Extracted proteins from treated and control cells were analysed using a comparative proteomic platform. Differentially expressed proteins, identified by the Panorama Xpress Profiler725 antibody microarray were submitted to Ingenuity Pathway Analysis. A total of 32 unique differentially expressed proteins were identified with a significant $(>1.8$-fold) difference in expression between treated and untreated cells in at least one cell line. Five molecules, BCL2L1 (Bcl-xL), BID, CHUK (IKK), FASLG and RAF1, were mapped to the Apoptosis Signaling pathway following Ingenuity Pathway Analysis. BCL2L1 (Bcl-xL) and BID were analysed using immunoblotting and differential expression was confirmed. Proteomic (antibody microarray) analysis suggests that the mechanism of action of DuP 697 may be exerted via the induction of apoptosis. The antibody microarray platform can be utilised to explore the molecular mechanism of action of novel anticancer agents.
\end{abstract}

\section{Introduction}

Malignant pleural mesothelioma (MPM) is an aggressive cancer affecting the pleura. Despite recent advances in chemotherapy,

Correspondence to: Dr Lynn Cawkwell, Research Laboratories, Daisy Building, Castle Hill Hospital, Hull HU16 5JQ, UK

E-mail: 1.cawkwell@hull.ac.uk

*Contributed equally

Abbreviations: COX-2, cyclooxygenase-2; DEP, differentially expressed protein; DMSO, dimethyl sulfoxide; HUVECs, human umbilical vein endothelial cells; IPA, Ingenuity Pathway Analysis; MPM, malignant pleural mesothelioma; tBID, truncated BID

Key words: cyclooxygenase-2, DuP 697, mesothelioma, antibody microarray, proteomics the median survival remains at approximately 12 months and the exploration of novel targets for therapeutic intervention is required. Cyclooxygenase-2 (COX-2) is an inducible enzyme which catalyses the conversion of arachidonic acid to prostaglandins in response to proinflammatory or mitogenic signals. It is overexpressed in many solid tumours and is a potential target for therapeutic intervention (1-4). Inhibition of COX-2 has been shown to have a significant anti-neoplastic effect by reducing the production of prostaglandins $(4,5)$. Our previous work demonstrated that COX-2 is overexpressed in 59\% (51/86) of archival malignant pleural mesothelioma tissue samples, a finding supported by similar studies (6-10). The cytotoxic effect of COX-2 inhibitors has been demonstrated in mesothelioma cell lines $(8,11)$ and recently we reported that specific COX-2 inhibitors, including DuP 697, induce anti-proliferative effects in mesothelioma cell lines (12). Several COX-2 inhibitors which are currently used in clinical practice, including celecoxib and rofecoxib, are derived from DuP 697 (13).

The chemotherapy options for patients with MPM are limited and improvements in survival are required. Pemetrexed, in combination with cisplatin, has been approved for first line chemotherapy in patients with MPM $(14,15)$. In mesothelioma cells, we have demonstrated that the cytotoxic effect of pemetrexed chemotherapy can be enhanced by the addition of DuP 697 (12). This compound is therefore worthy of further clinical investigation, however, the molecular mechanism of action of DuP 697 has not been widely studied. In normal proliferating human umbilical vein endothelial cells (HUVECs) expressing low levels of COX-2, DuP 697 was shown to induce apoptosis and this was associated with the upregulation of caspases 3, 8 and 9 (16). In the K562 chronic myeloid leukaemia cell line, DuP 697 induced G1-S cell cycle arrest and apoptosis with upregulation of caspase 8 (17). These hypothesis-driven studies suggest that the mechanism of cytotoxic action of DuP 697 may be via induction of apoptosis. We aimed to explore, using a novel proteomic platform, the molecular mechanism of action of this compound using cell lines derived from solid tumours.

\section{Materials and methods}

Cell line treatments. DuP 697 was previously demonstrated to have a cytotoxic effect in the COX-2 positive mesothelioma cell line MSTO-211H and in the lung cancer cell line A549, which 
was originally selected as a COX-2 positive cell line (12). In order to induce a visible cytotoxic effect in DuP 697 treated cells (50\% reduction in cell numbers compared to control cells treated with drug carrier only), MSTO-211H and A549 cells were treated with $31.7 \mu \mathrm{M}$ and $50 \mu \mathrm{M}$ DuP 697 (\#1430, Tocris Bioscience) respectively, for $72 \mathrm{~h}$. Drug carrier (dimethyl sulfoxide; DMSO) only was added to control cells. At the end of $72 \mathrm{~h}$ total protein lysates were generated from DuP 697 treated and control cells, using both antibody microarray buffer and immunoblotting buffer to yield at least $1 \mathrm{mg}$ of protein with a concentration of $1 \mathrm{mg} / \mathrm{ml}$.

Antibody microarray analysis. The Panorama Xpress Profiler725 antibody microarray kit (\#XP725, Sigma Aldrich), which consists of 725 antibodies spotted in duplicate on a nitrocellulose-coated glass microscope slide, was used for proteomic analysis as previously described (18). Total protein lysates from control (drug carrier only) samples were fluorescently labelled with Cy3 (\#PA23001, GE Healthcare) and lysates from DuP 697 treated samples were labelled with Cy5 (\#PA25001, GE Healthcare). Dye-to-protein molar ratios of at least 2 were achieved prior to protein binding. Equal amounts of protein from each sample were incubated with the microarray slide for 45 min on an orbital shaker. Normalisation and data analysis were performed as previously described and in all experiments the 'substances matched' value achieved was at least $90 \%$ (18). Differentially expressed proteins (DEPs) were considered significant with a fold change $\geq 1.8$, whilst fold changes $\geq 1.5$ were also recorded for each experiment for use as supporting data (18).

Ingenuity Pathway Analysis. Gene identifiers which corresponded to the DEPs were identified from the Ingenuity ${ }^{\circledR}$ Knowledge Base and the dataset was analysed through the use of Ingenuity Pathway Analysis (IPA; Ingenuity ${ }^{\circledR}$ Systems, www.ingenuity.com). The dataset containing gene identifiers of the DEPs was uploaded into the application and each identifier was mapped to its corresponding object in the Ingenuity ${ }^{\circledR}$ Knowledge Base. Canonical Pathways Analysis was used to identify pathways from the IPA library that were most significant to the dataset.

Semi-quantitative immunoblotting. Proteins were extracted in Laemmli buffer [62.5 mM Tris-HCl (pH 6.8), $10 \%$ glycerol, $2 \%$ SDS, $5 \% \beta$-mercaptoethanol, $1 \%$ protease inhibitor mix and $0.00125 \%$ bromophenol blue] and $20 \mu \mathrm{g}$ was electrophoresed on a $12 \%$ Precise gel (\#25222, Pierce) at a constant voltage of $140 \mathrm{~V}$ for $40 \mathrm{~min}$. Proteins were transferred using the iBlot dry transfer system (\#IB3010-01, Invitrogen) onto nitrocellulose membrane. The membrane was blocked in 5\% non-fat dry milk dissolved in Tris-buffered saline containing $0.05 \%$ Tween-20. A primary antibody against BCL2L1 (Bcl-xL; \#B9429, Sigma Aldrich) was applied at 1:5000 for $2 \mathrm{~h}$. A primary antibody against BID (\#ab32060, Abcam) was applied at 1:300 for $16 \mathrm{~h}$. As loading control, a primary antibody against alpha tubulin (\#ab7291, Abcam) was applied at 1:2500 for $2 \mathrm{~h}$. The relevant secondary antibody (\#SC-2030 or \#SC-2031, Santa Cruz Biotechnology) was applied at 1:1000 for $1 \mathrm{~h}$ and bands were detected using the Supersignal West Pico Chemiluminscent Substrate Kit (\#34078, Pierce). Films were scanned using a GS800 calibrated
Table I. A total of 32 unique DEPs identified using antibody microarray analysis following DuP 697 treatment of MSTO$211 \mathrm{H}$ mesothelioma cells and A549 lung cancer cells. ${ }^{a}$

\begin{tabular}{|c|c|c|c|c|}
\hline $\begin{array}{l}\text { Ab \# } \\
\text { (Sigma } \\
\text { Aldrich) }\end{array}$ & $\begin{array}{l}\text { Protein } \\
\text { target }\end{array}$ & $\begin{array}{l}\text { Gene } \\
\text { identifier }\end{array}$ & A549 & $\begin{array}{r}\text { MSTO- } \\
211 \mathrm{H}\end{array}$ \\
\hline P0084 & Pinin & PNN & 7.67 & 7.32 \\
\hline Z0377 & Zxyin & ZYX & 4.39 & 4.74 \\
\hline C1862 & Coilin & COIL & 4.6 & 3.46 \\
\hline A5968 & AP-1 & JUN & 2.49 & 2.97 \\
\hline B3183 & $\underline{\mathrm{tBID}}$ & $\underline{\mathrm{BID}}$ & 2.42 & 2.54 \\
\hline C7736 & Centrin & CETN1 & 2.51 & 2.04 \\
\hline S5446 & SUMO-1 & SUMO1 & 2.44 & 2.09 \\
\hline C6219 & Connexin-43 & GJA1 & 2.44 & 2.01 \\
\hline M0445 & MDMX & MDM4 & 2.13 & 2.39 \\
\hline A0844 & AP-2a & TFAP2A & 2.37 & 2.28 \\
\hline A7107 & AP2 & TFAP2A & 2.06 & 2.04 \\
\hline I6139 & IKKa & CHUK & 2.37 & 1.99 \\
\hline E8526 & $\mathrm{E} 2 \mathrm{~F} 4$ & $\mathrm{E} 2 \mathrm{~F} 4$ & 2.37 & 1.96 \\
\hline S1190 & SLIPR/MAGI-3 & MAGI3 & 2.15 & 1.92 \\
\hline B9429 & $\underline{B c l-x L}$ & $\underline{\text { BCL2L1 }}$ & 2.13 & 2.24 \\
\hline S9809 & $\mathrm{Sp} 1$ & SP1 & 2.13 & 1.95 \\
\hline F3648 & Fibronectin & FN1 & 1.99 & 2.12 \\
\hline F2051 & Fas ligand & FASLG & 1.81 & 2.14 \\
\hline V7881 & Vitronectin & VTN & 1.99 & 1.82 \\
\hline H9912 & Hsnf5/INI1 & SMARCB1 & 1.96 & 1.84 \\
\hline A7833 & ATF-1 & ATF1 & 1.83 & 1.84 \\
\hline $\mathrm{R} 8274$ & $\begin{array}{l}\text { RIP receptor } \\
\text { interacting protein }\end{array}$ & RIPK1 & 2.11 & 1.74 \\
\hline T5942 & $14-3-3$ theta/tau & YWHAQ & 1.64 & 2.22 \\
\hline $\mathrm{T} 1075$ & Tal & LRSAM1 & 2.05 & 1.72 \\
\hline C3470 & Connexin-32 & GJB1 & 2.04 & 1.76 \\
\hline R1151 & c-Raf pSer621 & RAF1 & 1.78 & 1.99 \\
\hline R4904 & Reelin & RELN & 1.95 & 1.67 \\
\hline S3934 & Smad4 (DPC4) & SMAD4 & 1.87 & 1.61 \\
\hline R3529 & Rnase L & RNASEL & 1.59 & 1.82 \\
\hline A5044 & Alpha actinin & ACTN1 & 1.82 & 1.66 \\
\hline E8767 & c-erbB-3 & ERBB3 & 1.82 & - \\
\hline C3956 & c-Myc & MYC & 1.81 & - \\
\hline L1538 & LIN-7 & LIN7A & 1.8 & 1.57 \\
\hline
\end{tabular}

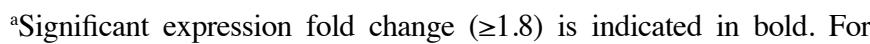
proteins which show $\geq 1.8$-fold change in expression in one cell line, supporting data from the second cell line is shown upward of 1.5-fold and non-significant values (below 1.5-fold) are indicated '-'. Underlined protein targets were selected for immunoblotting analysis.

densitometer (Bio-Rad) with Quantity One software (Bio-Rad). Following data normalisation against the loading control, differential expression between samples was calculated. 
Apoptosis Signaling

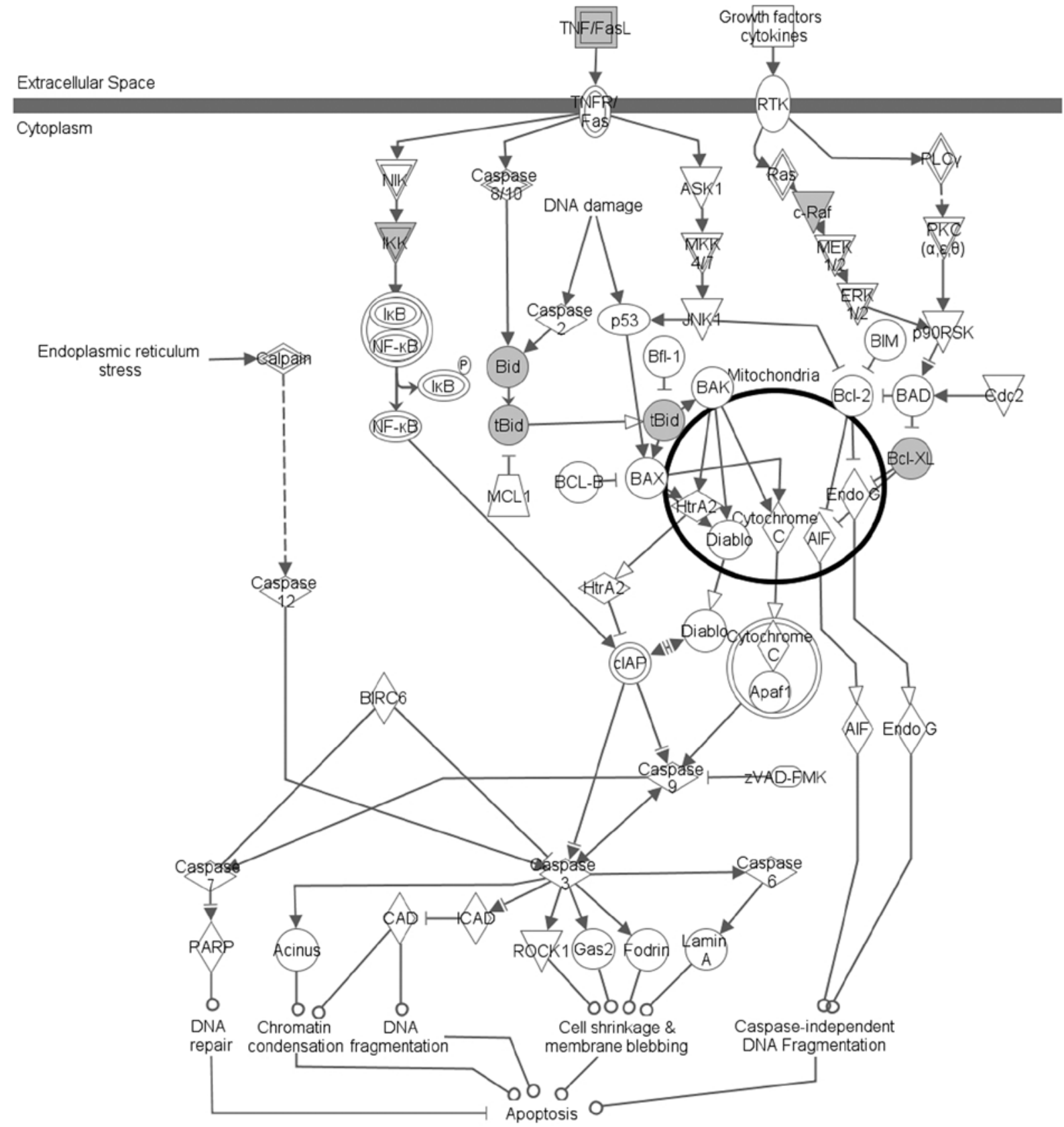

๑) 2000-2011 Ingenuity Systems, Inc. All rights reserved.

Figure 1. Apoptosis Signaling canonical pathway from IPA (Ingenuity ${ }^{\circledast}$ Systems, www.ingenuity.com) showing BCL2L1 (Bcl-xL), BID, CHUK (IKK), FASLG and RAF1 which were identified by antibody microarray analysis.

\section{Results}

Antibody microarray analysis identified 32 unique proteins which demonstrated $\geq 1$.8-fold difference in expression in at least one cell line, when comparing DuP 697 treated versus control (drug carrier only) cells (Table I). Of these, 20 DEPs demonstrated $\geq 1$.8-fold difference in $2 / 2$ cell lines. The dataset of 32 DEPs was submitted to IPA and the top relevant canonical pathway was 'Apoptosis Signaling', which involved 5 DEPs: BCL2L1 (Bcl-xL), BID, CHUK (IKK), FASLG and RAF1 (Fig. 1). The BCL2L1 (Bcl-xL) and BID proteins were selected for further analysis using immunoblotting. The anti-apoptotic BCL2L1 (Bcl-xL) protein was down-regulated by 2.48-fold in the MSTO-211H cell line when treated with DuP 697 (Fig. 2). The anti-tBID antibody (B3183), which was present on the antibody microarray, proved to be unreliable in the immunoblotting application. However, full length BID was found to be down-regulated in both the MSTO$211 \mathrm{H}$ and A549 cell lines by a fold change of 10.16 and 14.52 respectively, following treatment with DuP 697 (Fig. 3).

\section{Discussion}

We have previously confirmed that COX-2 is overexpressed in MPM samples which suggests that novel anticancer therapies 


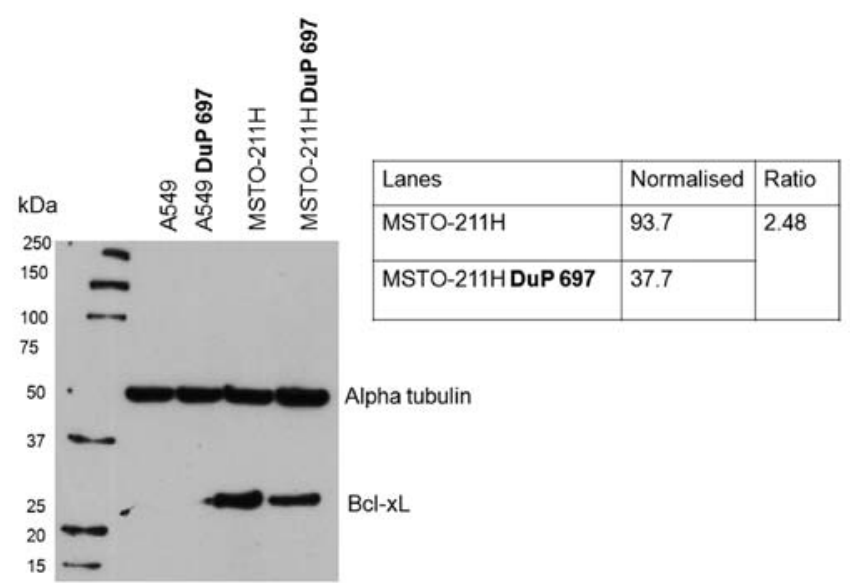

Figure 2. Immunoblotting demonstrated a significant decrease (2.48-fold) in expression of BCL2L1 (Bcl-xL) in the MSTO-211H mesothelioma cell line following treatment with DuP 697. The BCL2L1 (Bcl-xL) protein could not be detected in the A549 cell line. The primary antibody against BCL2L1 (Bcl-xL) used here (\#B9429, Sigma Aldrich) is expected to detect a band size of approximately $27 \mathrm{kDa}$. Alpha tubulin (\#ab7291, Abcam) is included as a loading control.

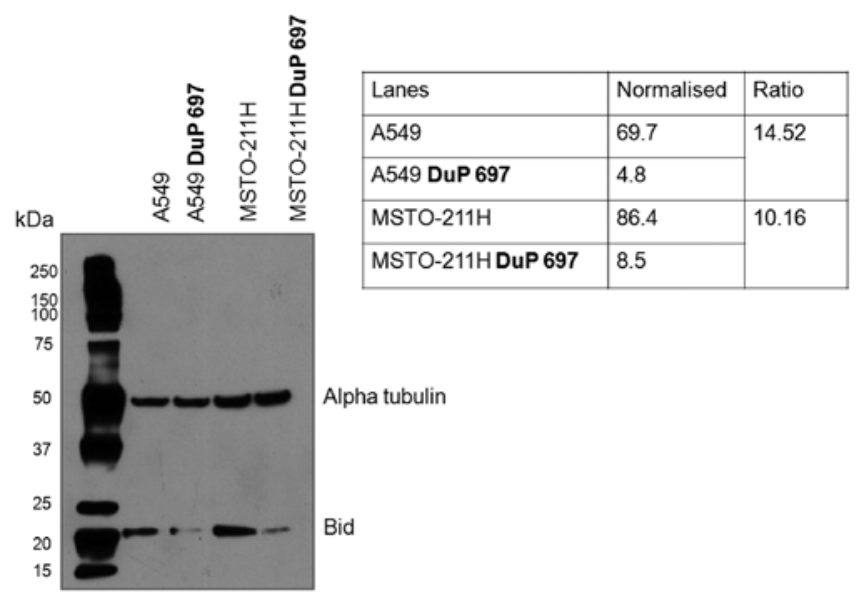

Figure 3. Immunoblotting demonstrated a significant decrease in expression of full length BID in the MSTO-211H mesothelioma and A549 lung cancer cell lines following treatment with DuP 697. The primary antibody against full length BID used here (\#ab32060, Abcam) is expected to detect a band size of approximately $22 \mathrm{kDa}$. Alpha tubulin (\#ab7291, Abcam) is included as a loading control.

targeted at this pathway may be useful in mesothelioma patients (10). In addition, we have demonstrated that the COX-2 inhibitor DuP 697 enhanced the cytotoxic effect of pemetrexed in mesothelioma cell lines, including MSTO-211H (12). It is important to understand the molecular mechanism of action of novel agents before possible clinical testing and DuP 697 has not been widely researched. In the present study we have explored the molecular mechanism of action of DuP 697 using an antibody microarray proteomic platform. We have identified 32 unique DEPs which were associated with DuP 697 treatment for $72 \mathrm{~h}$. Of these, 20 proteins demonstrated significant ( $\geq 1.8$-fold) differential expression in both the MSTO-211H mesothelioma and A549 lung cancer cell lines. Using some of the data from these, and other, experiments we have recently described Zyxin as the commonest repeatedly identified DEP (RIDEP) when using this proteomic platform (18) and therefore the selection of proteins for further analysis must be carefully considered. The analysis of the 32 DEPs using IPA indicated that 5 proteins, BCL2L1 (Bcl-xL), BID, CHUK (IKK), FASLG and RAF1, were associated with the Apoptosis Signaling canonical pathway. Following a positive signal for apoptosis, activated caspase 8 cleaves inactive, cytosolic, full length BID into active truncated BID (tBID), which localises to the mitochondrial membrane (19-21). The anti-apoptotic proteins BCL-2 and BCL2L1 (Bcl-xL) block the escape of cytochrome $\mathrm{C}$ from the mitochondria, by preventing Bax from forming channels in the mitochondrial membrane, until activated tBID is localised to the membrane (19-21). The onset of apoptosis may be associated with decreased levels of full length BID, due to its cleavage into tBID, and decreased levels of the anti-apoptotic protein BCL2L1 (Bcl-xL). Our immunoblotting data would support these suggested protein changes following administration of DuP 697 for $72 \mathrm{~h}$.

The caspase pathway of apoptosis has previously been implicated as the in vitro mechanism of action for DuP 697, with upregulation of caspases 3, 8 and 9 being observed in hypothesis-driven experiments in normal proliferating endothelial cells or leukaemia cells $(16,17)$. At the 72 -h time-point, which we examined here, we did not identify differential expression of caspases $3,4,5,6,7,8,9,10,11,12$ or 13 or pro-caspase 8 in either MSTO-211H or A549 cells. However, this may be due to the return of these proteins to basal levels within $72 \mathrm{~h}$ since the upregulation of caspases 3,8 and 9 was noted within $8 \mathrm{~h}$ in HUVECs (16).

COX-2 is a key enzyme involved in the metabolism of arachidonic acid resulting in the production of prostaglandins, particularly PGE2, which plays an important role in tumour progression. COX-2 inhibitors may act by inhibition of COX-2, but the exact mechanism of how COX-2 inhibitors exert an antineoplastic effect is currently unknown. Indeed, several studies have suggested that COX-2 inhibitors may act independently of COX-2 (22-25). In our antibody microarray experiments, differential expression of COX-2 was not observed in either cell line after treatment with DuP 697 for the duration selected (72 h). In future work, the expression of COX-2 and the individual proteins within the apoptosis signalling pathway, which we have implicated here, could now be examined over a timecourse of treatment with DuP 697.

We have demonstrated that the antibody microarray proteomic platform can be used to explore the molecular mechanism of a COX-2 inhibitor. This will prove useful in gaining a more thorough understanding of novel agents which may have clinical applications. Specific COX-2 inhibitors, such as DuP 697, may have a future therapeutic role in MPM. Our proteomic analysis suggests that the anti-proliferative effect of DuP 697, which was previously seen in mesothelioma cell lines, may be exerted via the induction of apoptosis. DuP 697, or other COX-2 inhibitors such as celecoxib or rofecoxib, may act as an effective apoptosis sensitiser when combined with chemotherapy drugs such as Pemetrexed and further studies are required to test this hypothesis.

\section{Acknowledgements}

This study was kindly supported by Yorkshire Cancer Research. The sponsor had no role in study design; collection, 
interpretation or analysis of data; writing of the manuscript; or decision to submit the article for publication.

\section{References}

1. Hull MA: Cyclooxygenase-2: how good is it as a target for cancer chemoprevention? Eur J Cancer 41: 1854-1863, 2005.

2. Gasparini G, Longo R, Sarmiento R and Morabito A: Inhibitors of cyclo-oxygenase 2: a new class of anticancer agents? Lancet Oncol 4: 605-615, 2003

3. Rao P and Knaus E: Evolution of nonsteroidal antiinflammatory drugs (NSAIDS): cyclooxygenase (COX) inhibition and beyond. J Pharm Pharm Sci 11: 81S-110S, 2008.

4. Ghosh N, Chaki R, Mandal V and Mandal SC: COX-2 as a target for cancer chemotherapy. Pharmacol Rep 62: 233-244, 2010

5. Menter DG, Schilsky RL and DuBois RN: Cyclooxygenase-2 and cancer treatment: understanding the risk should be worth the reward. Clin Cancer Res 16: 1384-1390, 2010.

6. Baldi A, Santini D, Vasaturo F, et al: Prognostic significance of cyclooxygenase-2 (COX-2) and expression of cell cycle inhibitors p21 and p27 in human pleural malignant mesothelioma. Thorax 59: 428-433, 2004

7. Edwards JG, Faux SP, Plummer SM, et al: Cyclooxygenase-2 expression is a novel prognostic factor in malignant mesothelioma Clin Cancer Res 8: 1857-1862, 2002.

8. Marrogi A, Pass HI, Khan M, Metheny-Barlow LJ, Harris CC and Gerwin BI: Human mesothelioma samples overexpress both cyclooxygenase-2 (COX-2) and inducible nitric oxide synthase (NOS2): in vitro antiproliferative effects of a COX-2 inhibitor Cancer Res 60: 3696-3700, 2000.

9. Cardillo I, Spugnini EP, Verdina A, Galati R, Citro G and Baldi A: Cox and mesothelioma: an overview. Histol Histopathol 20 1267-1274, 2005.

10. O'Kane SL, Cawkwell L, Campbell A and Lind MJ: Cyclooxygenase- 2 expression predicts survival in malignant pleural mesothelioma. Eur J Cancer 41: 1645-1648, 2005.

11. Catalano A, Graciotti L, Rinaldi L, et al: Preclinical evaluation of the nonsteroidal anti-inflammatory agent celecoxib on malignant mesothelioma chemoprevention. Int J Cancer 109: 322-328, 2004.

12. O'Kane SL, Eagle GL, Greenman J, Lind MJ and Cawkwell L: COX-2 specific inhibitors enhance the cytotoxic effects of pemetrexed in mesothelioma cell lines. Lung Cancer 67: $160-165,2010$.
13. Blobaum AL and Marnett LJ: Structural and functional basis of cyclooxygenase inhibition. J Med Chem 50: 1425-1441, 2007.

14. Scagliotti GV, Parikh P, von Pawel J, et al: Phase III study comparing cisplatin plus gemcitabine with cisplatin plus pemetrexed in chemotherapy-naive patients with advanced-stage non-small-cell lung cancer. J Clin Oncol 26: 3543-3551, 2008.

15. Vogelzang NJ, Rusthoven JJ, Symanowski J, et al: Phase III study of pemetrexed in combination with cisplatin versus cisplatin alone in patients with malignant pleural mesothelioma. J Clin Oncol 21: 2636-2644, 2003

16. Churchman A, Baydoun AR and Hoffman R: Inhibition of angiogenic tubule formation and induction of apoptosis in human endothelial cells by the selective cyclooxygenase- 2 inhibitor 5-bromo-2-(4-fluorophenyl)-3-(methylsulfonyl) thiophene (DuP-697). Eur J Pharmacol 573: 176-183, 2007.

17. Peng HL, Zhang GS, Liu JH, Gong FJ and Li RJ: Dup-697, a specific COX-2 inhibitor, suppresses growth and induces apoptosis on K562 leukemia cells by cell-cycle arrest and caspase- 8 activation. Ann Hematol 87: 121-129, 2008.

18. Hodgkinson VC, Elfadl D, Drew PJ, Lind MJ and Cawkwell L: Repeatedly identified differentially expressed proteins (RIDEPs) from antibody microarray proteomic analysis. J Proteomics 74: 698-703, 2011.

19. Danial NN: BCL-2 family proteins: critical checkpoints of apoptotic cell death. Clin Cancer Res 13: 7254-7263, 2007.

20. Song G, Chen G, Hu T and Lai PB: Bid stands at the crossroad of stress-response pathways. Curr Cancer Drug Targets 10: 584-592, 2010.

21. Strasser A, Cory S and Adams JM: Deciphering the rules of programmed cell death to improve therapy of cancer and other diseases. EMBO J 30: 3667-3683, 2011.

22. Kern MA, Haugg AM, Koch AF, et al: Cyclooxygenase-2 inhibition induces apoptosis signaling via death receptors and mitochondria in hepatocellular carcinoma. Cancer Res 66: 7059-7066, 2006.

23. Lou J, Fatima N, Xiao Z, et al: Proteomic profiling identifies cyclooxygenase-2-independent global proteomic changes by celecoxib in colorectal cancer cells. Cancer Epidemiol Biomarkers Prev 15: 1598-1606, 2006.

24. Pang RP, Zhou JG, Zeng ZR, et al: Celecoxib induces apoptosis in COX-2 deficient human gastric cancer cells through Akt/ GSK3beta/NAG-1 pathway. Cancer Lett 251: 268-277, 2007.

25. Schonthal AH: Direct non-cyclooxygenase-2 targets of celecoxib and their potential relevance for cancer therapy. Br J Cancer 97: 1465-1468, 2007. 